\title{
HACIA LA CALIFICACIÓN COMO ENTIDAD ASOCIATIVA \\ PRIORITARIA: LAS DIFERENTES FORMAS DE INTEGRACIÓN DE LAS COOPERATIVAS AGROALIMENTARIAS
}

\author{
POR \\ Cristina CANO ORTEGA ${ }^{1}$
}

\section{RESUMEN}

La Ley 13/2013, de 2 de agosto, de fomento de la integración de cooperativas y de otras entidades asociativas de carácter agroalimentario ha creado una nueva figura, la "Entidad Asociativa Prioritaria", cuyos requisitos para su reconocimiento han sido concretados recientemente a través del desarrollo reglamentario efectuado por el Real Decreto 550/2014, de 27 de junio. Esta ley trata de promover el aumento de la dimensión de las cooperativas agroalimentarias para que sean más competitivas, puesto que sufren de una evidente atomización, sobre todo, si se comparan con las cooperativas de este sector en el norte de Europa. Para ello la Ley 13/2013 se vale del incentivo de la concesión de una situación preferencial en las ayudas de los Programas de Desarrollo Rural. En primer lugar realizaremos un análisis de los distintos requisitos que se exigen para poder obtener la calificación como "Entidad Asociativa Prioritaria", como por ejemplo, el carácter supraautonómico de la entidad resultante, superar una determinada cifra de volumen de negocios, etc. Y, en segundo lugar, analizaremos las diversas vías de integración que pueden utilizarse para poder alcanzar la dimensión necesaria para ser reconocidas como "Entidad Asociativa Prioritaria".

\footnotetext{
${ }^{1}$ Dirección de correo electrónico: cristinacano@ual.es Estudio realizado en el marco del Proyecto de Investigación de Excelencia en Equipos de Investigación P12SEJ-2555, de la Consejería de Innovación, Ciencia y Empresa de la Junta de Andalucía, sobre "Retos y Oportunidades en los procesos de concentración e integración del sector agroalimentario"; del Grupo de Investigación de la Universidad de Almería SEJ-200, "Derecho Público y Privado de la Agroalimentación y de la Innovación Tecnológica", adscrito al Campus de Excelencia Internacional Agroalimentario (CEIA3); y del Seminario sobre Propiedad Industrial y Derecho de la Competencia de la Universidad de Almería.

REVESCO No 118 - Segundo Cuatrimestre 2015 - ISSN: 1885-8031 - www.ucm.es/info/revesco

http://dx.doi.org/10.5209/rev_REVE.2015.n118.49064
}

Fecha de recepción: 12/12/2014

Fecha de aceptación: 01/02/2015 
Palabras clave: fusión, grupos cooperativos, cooperativas de segundo grado, concentración y medidas públicas de fomento.

Claves Econlit: P13; Q13; K2; H2.

\title{
TOWARDS TO QUALIFICATION AS ASSOCIATIVE PRIORITY ENTITY: DIFFERENT FORMS OF INTEGRATION OF AGRI-FOOD COOPERATIVES
}

\begin{abstract}
The Act 13/2013, of $2^{\text {nd }}$ of August, to promote the integration of cooperatives and other associative agri-food entities has created a new figure, the "Associative Priority Entity" whose requirements for recognition have been recently concretized through regulatory development carried out by the Royal Decree 550/2014, of $27^{\text {th }}$ of June. This law seeks to promote the size increase of agri-cooperatives in order to be more competitive, due to the fact that they suffer from an obvious atomization, specially, if they are compared with the cooperatives of this sector in the Northern Europe. In order to achieve this aim, the Act $13 / 2013$ uses as an incentive the granting of a preferential situation in the Rural Development Programmes Aids. Firstly, it will be made an analysis of the requirements needed to be qualified as "Associative Priority Entity", such as the supra-regional nature of the resultant entity, reaching a certain threshold of turnover, etc. And, finally, it will be review the different ways of integration that can be used to achieve the necessary size to be recognized as an “Associative Priority Entity".
\end{abstract}

Keywords: merger, cooperative groups, second tier cooperatives, concentration and promotion public measures.

\section{INTRODUCCIÓN}

La Ley 13/2013, de 2 de agosto, de fomento de la integración de cooperativas y de otras entidades asociativas de carácter agroalimentario (en adelante, LFIC) ha creado una nueva figura, la "Entidad Asociativa Prioritaria". Con esta Ley se trata dar respuesta a la necesaria reestructuración a la que debe someterse el sector productivo agroalimentario español. En concreto, las cooperativas agroalimentarias tienen una dimensión muy reducida comparada con las cooperativas de este mismo sector en otros países de nuestro entorno. Por ello, se habla de un sector muy atomizado, que debe ganar dimensión para ser más competitivo, moderno, internacionalizado y que pueda aprovechar los beneficios ligados a un 
tamaño superior como son las economías de escala. La creación de la "Entidad Asociativa Prioritaria", como "pieza clave" de la Ley, responde a la búsqueda de una nueva figura que se forme con ciertos criterios objetivos, cualitativos y cuantitativos, que ayude a alcanzar la dimensión deseada. La consecuencia de obtener tal calificación es la de tener una situación preferente en las ayudas y subvenciones de los Programas de Desarrollo Rural, siempre dentro del respeto a la normativa de competencia, en concreto, a las normas sobre ayudas públicas. El Plan Estatal de Integración Asociativa debe establecer medidas que faciliten y favorezcan la integración de cooperativas.

A continuación se analizarán las diversas formas de integración que pueden utilizar las cooperativas agroalimentarias para conseguir la dimensión necesaria para ser consideradas como Entidad Asociativa Prioritaria. La ley menciona con frecuencia la fusión y, de una forma secundaria, se refiere a otras formas de "integración" de las cooperativas y demás formas asociativas. En concreto, la integración podrá hacerse creando una nueva entidad, desapareciendo las que la forman (fusión por constitución de nueva sociedad), o podrán desaparecer algunas para ser absorbidas por otra (fusión por absorción). También podrá ser posible que se reconozca como Entidad Asociativa Prioritaria a una entidad ya existente o que se cree una nueva entidad subsistiendo las entidades originales que la forman, constituyendo una entidad de grado superior que se encargue de las tareas de comercialización en común de la producción de todos los socios de dichas entidades (cooperativas de segundo grado). Además, la Ley menciona expresamente los grupos cooperativos conceptuándolos como aquéllos que asocian a varias cooperativas con la entidad de cabeza de grupo que ejercita facultades o emite instrucciones de obligado cumplimiento para las sociedades integrantes del grupo, caracterizándose por la unidad de decisión. Aunque existen otras vías de integración o asociación, nos limitaremos a estudiar la fusión, las cooperativas de segundo grado y los grupos cooperativos.

\section{LAS ENTIDADES ASOCIATIVAS PRIORITARIAS}

En el marco coyuntural de la actual crisis económica, el sector agroalimentario está respondiendo mejor que otros ante tal delicada situación. En parte, esto se debe a la innovación de ciertos subsectores y a la importancia de la exportación de productos, sobre todo de algunos como los hortofrutícolas. Aun así, se caracteriza porque el sector productor tiene una estructura atomizada, es decir, el modelo de la empresa agroalimentaria española es el de una empresa pequeña con pocos trabajadores y un volumen de negocios reducido, lo 
cual limita su poder de negociación frente a la industria y los distribuidores. Asimismo, esto dificulta la consecución de economías de escala que le permitan reducir costes y ofrecer productos más competitivos en el mercado, en el que predominan las empresas agroalimentarias de países de nuestro entorno, que tienen una gran dimensión.

Dando cumplimiento al mandato constitucional del artículo 129.2 de la Constitución española (CE) sobre el fomento de las sociedades cooperativas, la LFIC $^{2}$ es la respuesta dada por los poderes públicos a estos problemas. Esta norma pretende corregir los problemas de atomización de la oferta en el sector agroalimentario español ${ }^{3}$, que ha sido destacado reiteradamente en distintos informes de autoridades nacionales y europeas ${ }^{4}$. Para ello, fomenta la fusión e integración de las cooperativas agrarias y de otras formas asociativas en el sector agroalimentario, que tengan un ámbito de actuación superior al de una Comunidad Autónoma. En este sentido, se ha aprobado un Plan Estatal de Integración Asociativa ${ }^{5}$ que pretender coordinar las políticas enunciadas en la LFIC y eliminar los obstáculos que dificultan la integración, siempre dentro del marco y respeto del Derecho de la competencia. Por tanto, el objetivo de la Ley es el incremento del tamaño y escala de los productores agroalimentarios. El fomento de la integración en el sector agroalimentario, se promueve por la LFIC a través del reconocimiento de un derecho de preferencia en ayudas y subvenciones previstas en los Programas de Desarrollo Rural ${ }^{6}$, pero no a cualquier entidad del sector que realice o haya realizado algún proceso de integración, sino que se limita a aquéllas entidades

\footnotetext{
${ }^{2}$ La valoración realizada por la CNC y el Consejo Económico y Social sobre el Anteproyecto de la Ley, en general, fue positiva. Puede verse al respecto, CNC: IPN 82/12. Anteproyecto de Ley de Fomento de la Integración Cooperativa y Asociativa; y Consejo Económico y Social: Dictamen 4/2012 sobre el Anteproyecto de Ley de Fomento de la integración de cooperativas y de otras entidades asociativas de carácter agroalimentario, 2012, o puede consultarse un resumen en Consejo Económico y Social, 2013: 102-105. La opinión de la doctrina de esta norma ha sido generalmente también buena. Por ejemplo, Calvo Vérgez, 2013: 6 considera que la LFIC debería ayudar a reforzar el papel de las cooperativas en el sector agroalimentario, configurando "un modelo cooperativo empresarial, rentable, competitivo, profesionalizado, generador de valor y con una dimensión relevante, en aras de mejorar la competitividad y rentabilidad en el sector agroalimentario".

3 "El sector cooperativo está compuesto por cerca de 4.000 entidades" [Preámbulo (I) LFIC].

${ }^{4}$ Pueden verse, por ejemplo: Report on competition law enforcement and market monitoring activities by European competition authorities in the food sector (European Competition Network Subgroup Food), Mejorar el funcionamiento de la cadena alimentaria en Europa (Comunicación de la Comisión al Parlamento Europeo, al Consejo, al Comité Económico y Social Europeo y al Comité de las Regiones; el Dictamen del Comité Económico y Social Europeo sobre el mismo; y las Conclusiones de la Presidencia sobre dicha Comunicación), Cooperativas y desarrollo agroalimentario (Dictamen del Comité Económico y Social Europeo) e Informe sobre Competencia y Sector Agroalimentario (CNC).

${ }^{5}$ En enero de 2014 fue aprobado el Plan Estatal de Integración Asociativa para el ejercicio 2014, cuyos cinco ejes principales de actuación son: 1) incremento de dimensión y modernización de estructuras mediante la integración de entidades asociativas; 2) formación e información de órganos rectores y directivos; 3) internacionalización; 4) comercialización y orientación al mercado; y 5) seguimiento del desarrollo del cooperativismo y evaluación del plan estatal.

${ }^{6}$ Pueden tener distintas finalidades: inversiones para la mejora de la gestión, transformación y comercialización; para internacionalización; acceso a financiación, etc. (art. 4.1 a 3 LFIC).
} 
productivas que alcancen una determinada dimensión y obtengan la calificación de Entidad Asociativa Prioritaria (EAP), además de a las entidades asociativas que las integran y sus productores agrarios. Estas ayudas deben ser examinadas teniendo en cuenta la normativa comunitaria sobre ayudas de Estado, pues pueden afectar negativamente a la competencia. Tradicionalmente, los socios, los directivos y rectores de las cooperativas se han mostrado reticentes a integrar su sociedad, por temor a la pérdida de autonomía o de puestos de trabajo, por lo que sólo acuden a esta vía en casos de extrema necesidad, sobre todo, de carácter financiera. La LFIC trata de incentivar que los socios contribuyan y respalden este tipo de operaciones, mediante el aliciente de conseguir un tratamiento preferencial en las políticas públicas cuando su cooperativa alcance el reconocimiento de EAP.

El modelo de integración que se suele emplear depende del modelo general que se siga en cada región. En algunas Comunidades Autónomas, las cooperativas de primer grado de considerables dimensiones tienen una mayor implantación que otro tipo de modelos de integración ${ }^{7}$. Por el contrario, las cooperativas de algunas Comunidades Autónomas, por su limitada extensión territorial, se ven "obligadas" a ampliar su ámbito de actuación a las regiones limítrofes, normalmente a través de la constitución de cooperativas de segundo grado o grupos cooperativos, lo cual sucede con menos frecuencia en las cooperativas de Comunidades Autónomas de mayor tamaño como Andalucía, Castilla y León o Castilla-La Mancha. En cualquier caso, hay que tener en cuenta que no para todas las cooperativas es necesario alcanzar una gran dimensión, pues en determinados sectores las cooperativas están bien organizadas y gestionadas, siendo muy competitivas, o en un determinado sector existen cupos o cuotas que limitan las producciones.

La concreción de los requisitos necesarios para el reconocimiento de las EAP se dejó al desarrollo reglamentario, lo cual ha sido llevado a efecto en el reciente Real Decreto $550 / 2014$, de 27 de junio, por el que se desarrollan los requisitos y el procedimiento para el reconocimiento de las Entidades Asociativas Prioritarias y para su inscripción y baja en el Registro Nacional de Entidades Asociativas Prioritarias, previsto en la Ley 13/2013, de 2 de agosto, de fomento de la integración de cooperativas y de otras entidades asociativas de carácter agroalimentario (en adelante, REAP). Como del propio título de la norma citada se deduce, ésta tiene por objeto determinar el umbral económico de facturación, según el sector productivo de que se trate, que se requiere alcanzar para poder tener la consideración de EAP;

\footnotetext{
${ }^{7}$ Por ejemplo, es el caso de las cooperativas de Castilla y León según el Plan de Acción en Cooperativas Agroalimentarias de Castilla y León 2014-2015, apartado 8.1.
} 
regular el procedimiento para el reconocimiento de EAP de carácter supra-autonómico; y regular el procedimiento para su inscripción en el Registro Nacional de Entidades Asociativas Prioritarias, que se creó por mandato de la LFIC.

El tipo de entidades asociativas agroalimentarias que pueden solicitar su reconocimiento como EAP son: 1) las sociedades cooperativas agroalimentarias; 2) las cooperativas de segundo grado; 3 ) los grupos cooperativos; 4) las sociedades agrarias de transformación; 5) las organizaciones de productores con personalidad jurídica propia, reconocidas de acuerdo con la normativa comunitaria en el ámbito de la Política Agraria Común $^{8}$; y 6) las entidades civiles o mercantiles -si son sociedades anónimas, sus acciones deberán ser nominativas-, siempre que más del 50 por ciento de su capital social pertenezca a sociedades cooperativas, a organizaciones de productores o a sociedades agrarias de transformación (art. 1.3 LFIC y art. 2.1 REAP). Es curioso como se ha equiparado a las SAT en el tratamiento dado a la integración de cooperativas, esto sin duda resultado de su cuestionada naturaleza que se debate entre la economía social y la del mercado (Vargas, 2012) y a su importancia en el sector. Pueden solicitar tal reconocimiento tanto las entidades ya existentes como aquellas que vayan a constituirse y cumplan los requisitos enunciados en la Ley y el Reglamento.

Para obtener la calificación de prioritaria, las entidades han de cumplir, además, otros requisitos (art. 3.1 LFIC, art. 2, apartados 2, 3 y 6 y art. 3.2 REAP) ${ }^{9}:$ 1) Tener implantación y un ámbito de actuación económico de carácter supra-autonómico. Se entiende que las entidades asociativas tienen un ámbito de actuación supra-autonómico si se dan simultáneamente estas dos circunstancias: a) tienen socios en más de una Comunidad Autónoma, con el límite de que no supere el 90 por ciento en el ámbito de una sola. En el supuesto de sociedades de capital, el porcentaje se determina exclusivamente respecto de las entidades integradas en ellas; y b) su actividad económica no supere el 90 por ciento en una sola Comunidad Autónoma. Estos porcentajes se elevarán al 95 por ciento si la producción del producto por el que solicita el reconocimiento como EAP se localiza en más de un 60 por ciento en una única Comunidad Autónoma o la entidad desarrolla su actividad en más de un

\footnotetext{
${ }^{8}$ La Comisión Nacional de la Competencia, en su informe sobre el Anteproyecto de Ley, no consideraba que las organizaciones de productores reconocidas de acuerdo con la normativa comunitaria en el marco de la PAC debieran incluirse como entidad asociativa prioritaria, entre otros motivos, porque no suponen necesariamente "la integración estable de las estructuras productivas de sus miembros". De igual forma, el Consejo Económico y Social (Consejo Económico y Social, 2013).

${ }^{9}$ El Gobierno de Cataluña ha interpuesto un recurso de inconstitucionalidad (núm. 6228-2013) contra los artículos 3, apartados 2 y 3, y 5 de la LFIC (BOE núm. 286 de 29 de noviembre de 2013).
} 
50 por ciento en una Comunidad Autónoma con cinco o más provincias (art. 2.5 REAP). 2) Realizar la comercialización conjunta de toda la producción de las entidades asociativas y de los productores que las componen. 3) La facturación de la entidad asociativa solicitante o la suma de las facturaciones de las entidades que se fusionan o integran alcancen determinada cifra. En concreto, si se encuentra en alguno de estos supuestos, cumpliéndolo en cualquiera de los tres últimos ejercicios económicos cerrados previos a la solicitud: a) si se solicita el reconocimiento para un producto determinado -se puede solicitar para varios-, su facturación anual, correspondiente a la producción comercializada de dicho producto, debe superar la cuantía señalada en el apartado a) del anexo I REAP, para la facturación total de la entidad; o b) si se solicita un reconocimiento genérico, su facturación total anual correspondiente al conjunto de productos comercializados por la entidad, debe superar la cuantía que se recoge en el apartado b) del anexo I REAP. Los valores indicados anteriormente se ven reducido un 30 por ciento si la entidad solicitante es una sociedad cooperativa agroalimentaria de primer grado. En la determinación del volumen anual de facturación se tiene en cuenta para las cooperativas agroalimentarias, en relación a las adquisiciones con terceros, el límite fijado en el 50 por ciento del total de las operaciones realizadas por la cooperativa (art. 93.4 LCOOP y art. 9.2 LRFC). 4) La constancia en los estatutos o disposiciones reguladoras correspondientes a las entidades que componen la EAP, además de en los de la propia entidad solicitante, de la obligación de los productores de entregar la totalidad de su producción, para su comercialización en común. 5) Los estatutos o disposiciones reguladoras de la EAP y de las entidades que la forman, deben contemplar las previsiones necesarias que aseguren a sus productores asociados el control democrático de su funcionamiento y de sus decisiones, asimismo, deben evitar la posición de dominio de uno o varios de sus miembros, y deberán respetar el principio de libre adhesión voluntaria y abierta cuando la entidad solicitante sea una cooperativa agroalimentaria. Además, deberá constar el consentimiento de los socios a la cesión de datos referentes a la actividad económica realizada en la entidad.

Al tratarse de una figura de ámbito supra-autonómico, será difícil que muchas entidades puedan optar a tal calificación, puesto que, debido a nuestro "curioso" y complicado marco normativo de las cooperativas, éstas suelen limitar su ámbito de actuación (al menos de forma principal, que es lo que determina la ley aplicable) a una sola Comunidad Autónoma. Por ello, las Comunidades Autónomas han comenzado a reaccionar y a ofrecer su homólogo de ámbito regional, las "Entidades Asociativas Agroalimentarias Prioritarias de carácter regional" (EAPr). La primera ha sido Castilla y León, que ha incorporado ya la figura tanto en 
la Ley 1/2014, de 17 de marzo, Agraria de Castilla y León (art. 158.2 y 3) como en el Plan de Acción del Cooperativismo 2014/2015. Igualmente, en el nuevo Programa de Desarrollo Rural 2014-2020 de Castilla y León está previsto conceder un tratamiento diferenciado a las EAPr, así como a los socios de estas entidades, agricultores y ganaderos ${ }^{10}$.

En cuanto al procedimiento de reconocimiento de las EAP, se iniciará por la solicitud del representante legal de la propia entidad o por los promotores de la misma si está en proceso de constitución, que irá dirigida al Director General de la Industria Alimentaria del Ministerio de Agricultura, Alimentación y Medio Ambiente, ya sea mediante presentación presencial o a través de la sede electrónica (art. 3.1 REAP). La solicitud debe ir acompañada por una serie de documentos ${ }^{11}$. En el caso de existir defectos en la solicitud o en la documentación que la acompaña, se requerirá al interesado para que subsane tales defectos en el plazo de 10 días, y en el caso de que no lo hiciera, se le tendrá por desistido de su petición (art. 4 REAP). La Dirección General de la Industria Alimentaria dictará resolución motivada y la notificará a la entidad solicitante en el plazo de seis meses. Si transcurrido este tiempo no se ha dictado resolución, se entenderá estimada la solicitud. Si la resolución es negativa podrá interponerse recurso de alzada ante la Secretaría General de Agricultura y Alimentación en el plazo de un mes. La resolución estimatoria queda condicionada a la efectiva constitución de la entidad, si esta se encontraba en constitución. Una vez estimada la solicitud, la Dirección General de la Industria Alimentaria procederá a la inscripción de oficio de la entidad en el Registro Nacional de Entidades Asociativas Prioritarias o una vez que se haya constituido ésta. Se remitirá copia de la hoja registral a los órganos competentes de las Comunidades Autónomas afectadas por el ámbito de la EAP para que tengan conocimiento de la inscripción

\footnotetext{
${ }^{10}$ El artículo 159 de la Ley Agraria de Castilla y León, da el mandato de poner en marcha iniciativas dirigidas a favorecer la integración de las cooperativas agrarias y de otras entidades de naturaleza asociativa como medio para lograr ciertos objetivos como incrementar la concentración de la oferta, agrupar los primeros eslabones de la cadena alimentaria, mejorar la formación y especialización de los equipos directivos y de gestión de dichas entidades y favorecer los procesos de transformación de los productos agrarios y mejorar su acceso a los mercados.

${ }^{11}$ En concreto, por el acuerdo de voluntades de la sociedad que pretende constituirse como EAP o del acuerdo del órgano de gobierno -si ya existía la entidad-, con los datos identificadores de la entidad; relación de todas las entidades que la integran en su caso, con la identificación de todos los productores que las componen y la indicación del territorio de la Comunidad Autónoma en la que llevan a cabo su actividad; los estatutos o disposiciones reguladoras de la entidad solicitante y de cada una de las que la componen con el contenido anteriormente comentado; la facturación de la entidad solicitante o la suma de las entidades que se integran y sus productores para los productos específicos o genéricos para los que se solicita el reconocimiento; y, por último, en el caso de que se acojan al periodo transitorio que reconoce la Disposición transitoria única REAP, según la cual tendrán un periodo máximo de cinco años para alcanzar la entrega total de los productos objeto de reconocimiento para su comercialización conjunta siguiendo el calendario previsto en el propio reglamento, deberán aportar la correspondiente declaración o compromiso de la entidad solicitante y de las que la integran (art. 3.2 REAP).
} 
y reconocimiento de la entidad (art. 5 REAP). Cada año, al cierre de su ejercicio económico, las EAP deberán presentar el depósito anual de la relación de productores que formen parte de la EAP y de las entidades que la integran; la memoria económica y social anual e informe auditor -en su caso-; y comunicación de toda modificación que afecte al reconocimiento como EAP, todo a efectos de actualización del Registro (art. 6 REAP).

Además, como hemos mencionado anteriormente, el REAP tiene por objeto (arts. 7 y 8) la regulación del Registro Nacional de Entidades Asociativas Prioritarias, que se encuentra adscrito a la Dirección General de la Industria Alimentaria del Ministerio de Agricultura, Alimentación y Medio Ambiente, que se encargará de la gestión, mantenimiento y actualización de las EAP inscritas. Cualquier modificación se realizará a instancia del interesado, y la baja en el Registro podrá producirse de oficio o a instancia del interesado. Los interesados deben instar la modificación de cualquier acto inscrito que no se adecue a la realidad en el plazo de un mes desde que ésta se produjera. Si tal modificación supusiera el incumplimiento sobrevenido de alguno de los requisitos del artículo 3.2 del Reglamento, supondrá la baja provisional en el Registro, durante un plazo de tres meses, y la baja definitiva, tras el oportuno procedimiento administrativo, si no se subsana en ese periodo. Las EAP también causarán baja en el Registro por voluntad expresa, por disolución o liquidación, por modificación de su ámbito territorial, o por incumplimiento de cualquiera de los requisitos exigidos por los artículos 3 y 5.4 de la LFIC o de los establecidos en el REAP. Asimismo, la Dirección General de la Industria Alimentaria procederá de oficio a la baja del registro de EAP de las entidades, que no presenten la memoria anual o incumplan la obligación de comunicar cualquier cambio que pueda afectar a su condición de prioritaria, tras el correspondiente apercibimiento, al que seguirá, en el plazo de tres meses, una resolución motivada, recurrible en el plazo de un mes.

Desde que se aprobara la LFIC y, sobre todo, el REAP, las cooperativas y las asociaciones representativas de las mismas han iniciado intensas negociaciones buscando la vía adecuada para que las cooperativas se integren y alcancen los requisitos establecidos para que puedan conseguir la calificación como $\mathrm{EAP}^{12}$. Las propias ventajas competitivas asociadas a una mayor dimensión deben ser el motor principal de este movimiento de integración, y no sólo realizar estas operaciones pensando en las ayudas y subvenciones que van a recibir las EAP como parece ocurrir en algunos casos. Son numerosas las cuestiones

\footnotetext{
${ }^{12}$ Por ejemplo, es el caso de OVISPAN formada por entidades del sector del ovino de diferentes CCAA (Extremadura, Aragón y Andalucía) que recientemente ha solicitado su reconocimiento como EAP.
} 
que deben estudiarse antes de proceder a la efectiva integración: con qué cooperativa integrarse, la fórmula de integración apropiada, el ámbito de actuación de las cooperativas implicadas, el volumen de negocios, etc. A continuación pasaremos a estudiar los diferentes procesos de integración a los que se refiere la LFIC.

\section{LOS DIFERENTES PROCESOS DE INTEGRACIÓN}

Los motivos para integrarse pueden ser muy variados: de índole económica, para ser más competitivos; seguir la tendencia de concentración empresarial del resto de sociedades; acceso a las nuevas tecnologías de la información y comunicación; la obtención de ventajas que conlleva un mayor tamaño en la empresa (economías de escala, acceso a fuentes de financiación, mayor poder de mercado, incremento del poder frente la competencia); defensa del movimiento cooperativo; etc. (Puentes, Velasco, Vilar, 2010). La LFIC menciona los diferentes procesos de integración de cooperativas (y otras entidades asociativas) que pueden ser utilizados con el fin de conseguir una mayor dimensión ${ }^{13}$. Parece incidir de forma especial en la fusión, aunque igualmente también incluye a las cooperativas de segundo grado y a los grupos cooperativos como formas de integración cooperativa ${ }^{14}$. En verdad, son estas dos últimas las formas genuinas de integración ${ }^{15}$ en el ámbito cooperativo, pues a la fusión se la

\footnotetext{
13 Algunas de las vías previstas por la LFIC para conseguir alcanzar una dimensión mayor pueden ser problemáticas para la libre competencia.

${ }^{14} \mathrm{La}$ "integración cooperativa" comprende también distintas modalidades de acuerdos intercooperativos como sociedades, agrupaciones, consorcios y uniones. Además, el término de integración también puede aplicarse a otras formas de colaboración con finalidades de organización representativa, de defensa y promoción de intereses como las asociaciones, uniones, federaciones y confederaciones de cooperativas ("asociacionismo cooperativo") (Sánchez, 2011). Los acuerdos intercooperativos permiten realizar a las cooperativas y sus socios operaciones de suministro, entregas de productos o servicios en la otra cooperativa firmante del acuerdo, teniendo la consideración de operaciones cooperativizadas con los propios socios (art. 79.3 LCOOP). No obstante, estos mecanismos de integración no han sido previstos por la LFIC, que sólo se refiere a entidades asociativas con personalidad jurídica propia. Los acuerdos entre empresas pueden ser considerados, en determinadas circunstancias, como prácticas colusorias que impiden, restringen o falsean de manera sensible la competencia en un mercado en concreto (arts. 1 LDC y 101 TFUE). Se trata de una materia escasamente tratada por la doctrina, aunque existen algunos valiosos estudios (Paz, 2004). También debe tenerse en cuenta el papel que juega la Sociedad Cooperativa Europea (en adelante, SCE) para la integración de cooperativas, por ejemplo, al permitir su constitución por cooperativas (cooperativa de segundo grado) o por su constitución mediante fusión de cooperativas. Sobre esta figura existen ya importantes aportaciones doctrinales (Vicent, 2003; Martínez, 2003; Lambea, 2005; Arcas, 2008; Pastor, 2009; Alfonso, 2010; o más recientemente, Vargas, 2015).

${ }^{15}$ Se considera que la concentración se refiere sólo a términos económicos, es decir, es idóneo para las empresas de capital cuya finalidad básica es la maximización de los beneficios. En cambio, "el término integración se refiere a la empresa cooperativa cuyos fines van más allá de los meramente económicos de maximización del beneficio, persiguiendo otros en los cuales priman términos como democracia o equidad frente a la eficiencia" (Puentes, Velasco, Vilar, 2010: 104).
} 
suele considerar como un proceso de concentración empresarial ${ }^{16}$ (Parra, 1974; o Embid, 1991).

\subsection{La fusión}

En el ámbito de las cooperativas, la fusión ha solido ceder protagonismo ante la transformación, modificación estructural por excelencia utilizada por las cooperativas en el momento en que las limitaciones de acceso a fuentes de capital o el deseo de los socios de poder hacer efectivo el verdadero valor de la sociedad, llevan a la conversión en otros tipos sociales. Los motivos del raro uso de la fusión por parte de las cooperativas se debe a: 1) El rendimiento económico de las cooperativas no está en proporción directa con la concentración e inversión en capital, sino con el incremento de la actividad cooperativizada de sus socios; 2) La propia naturaleza de las cooperativas como sociedades de capital variable y de libre adhesión o de puertas abiertas: la incorporación de más capital, más patrimonio, más socios y mayor presencia en el mercado, se realiza de forma casi natural; 3) El efecto perseguido con la fusión puede conseguirse con otras soluciones jurídicas de integración cooperativa: cooperativas de segundo grado, los grupos de cooperativas o los acuerdos intercooperativos (Macías, 2006).

La fusión supone el grado máximo de integración entre dos sociedades, ya que donde antes había dos o más sociedades independientes, tras las operación, sólo queda una (ya sea una de las preexistentes o de nueva creación), habiéndose integrado todos los patrimonios y todos los socios de todas las cooperativas en la sociedad resultante. El régimen jurídico previsto en las leyes cooperativas, tanto en la estatal como en la mayoría de las autonómicas, es muy similar al contemplado en la Ley 3/2009, de 3 de abril, sobre modificaciones estructurales de las sociedades mercantiles (LME). Esto se debe a la inevitable influencia ejercida por la Ley de Sociedades Anónimas (LSA) y al efecto armonizador de las Directivas Comunitarias en materia de fusión de sociedades.

\footnotetext{
${ }^{16}$ La fusión es uno de los medios de concentración de empresas, que si supera ciertos límites cuantitativos debe ser comunicada y controlada por la autoridad de la competencia estatal o comunitaria, según la dimensión de la operación (arts. 7 y ss. LDC y Reglamento del Consejo n 139/2004, de 20 de enero de 2004, sobre el control de las concentraciones entre empresas), puesto que con la concentración se puede crear o reforzar una posición de dominio que obstaculice significativamente la competencia en el mercado (Calvo, Carrascosa, 2003). La concentración de empresas supone un cambio en la estructura del mercado en el que opera la empresa al reducirse el número de competidores, lo que puede implicar un perjuicio para el consumidor que ve disminuir la variedad de productos y precios ofertados.
} 
En nuestro ordenamiento jurídico coexisten una regulación estatal ${ }^{17}$ y una regulación autonómica de las cooperativas ${ }^{18}$. Conforme al Bloque de constitucionalidad, la competencia sobre el régimen de cooperativas queda integrada dentro de las competencias autonómicas ${ }^{19}$. Cada Comunidad Autónoma, a excepción de Canarias que aún está en fase de aprobación, tiene una ley de cooperativas propia. Estas sociedades se someten a un régimen jurídico diverso dependiendo de dónde desarrollen su actividad ${ }^{20}$. Aunque esta riqueza normativa puede ser positiva en determinados aspectos, complican los procesos de integración supraautonómicos. Existen diferentes modelos de regulación de la fusión. Algunos dedican unos pocos artículos a ofrecer alguna particularidad procedimental de esta operación (por ejemplo, la LFCN), en cambio otros, desarrollan minuciosamente el régimen jurídico de ésta (por ejemplo, la LCCM). Aun así, el procedimiento de fusión y los mecanismos de protección de acreedores y socios están configurados de una forma muy similar en todas las normas ${ }^{21}$. Existen pocas diferencias, limitándose sobre todo al destino de los fondos irrepartibles en el caso de fusión heterogénea, el plazo de ejercicio del derecho de oposición o de separación, la fecha máxima de los balances de fusión, etc. Aunque una regulación extensa no siempre es la mejor opción, puesto que puede dotar de un marco jurídico inflexible a estas operaciones, tampoco es aconsejable un régimen demasiado indeterminado o abierto, ya que es necesario que el procedimiento se revista de una serie de garantías en beneficio de los socios y acreedores sociales.

\footnotetext{
${ }^{17}$ Ley 27/1999, de 16 de julio, de Cooperativas (LCOOP). Además contamos con la Ley 3/2011, de 4 de marzo, de la Sociedad Cooperativa Europea con domicilio en España.

${ }^{18}$ Ordenadas por orden cronológico son: Ley 4/1993, de 24 de junio, de Cooperativas del País Vasco (LCPV); Ley 2/1998, de 26 de marzo, de Sociedades Cooperativas de Extremadura (LSCExt.); Ley 5/1998, de 18 de diciembre, de Cooperativas de Galicia (LCG); Ley 4/1999, de 30 de marzo, de Cooperativas de Madrid (LCCM); Ley 4/2001, de 2 de julio, de Cooperativas de la Rioja (LCLR); Ley 4/2002, de 11 de abril, de Cooperativas de Castilla y León (LCCYL); Ley 18/2002, de 5 de julio, de Cooperativas de Cataluña (LCCAT); Ley 1/2003, de 20 de marzo, de Cooperativas de Islas Baleares (LCIB); Ley 8/2003, de 24 de marzo, de Cooperativas de Comunidad Valenciana (LCCV); Ley 8/2006, de 16 de noviembre, de Sociedades Cooperativas de la Región de Murcia (LSCRM); Ley Foral 14/2006, de 11 de diciembre, de Cooperativas de Navarra (LFCN); Ley 4/2010, de 29 de junio, de Cooperativas del Principado de Asturias (LCPA); Ley 11/2010, de 4 de noviembre, de Cooperativas de Castilla-La Mancha (LCCLM); Ley 14/2011, de 23 de diciembre, de Sociedades Cooperativas Andaluzas (LCAND); Ley 6/2013, de 6 de noviembre, de Cooperativas de Cantabria (LCCANT) y Decreto Legislativo 2/2004, de 29 de agosto, del Gobierno de Aragón, por el que se aprueba el texto refundido de la Ley de Cooperativas de Aragón (LCARA).

${ }^{19}$ En la lista de competencias exclusivas del Estado del artículo 149.1 no se hace referencia alguna a las cooperativas. Además, la Sentencia del Tribunal Constitucional 72/1983 también lo considera una competencia autonómica (León Sanz, 1997).

${ }^{20}$ Ese es el criterio delimitador entre la Ley de Cooperativas estatal y las autonómicas: si desarrollan su actividad de forma principal en una determinada Comunidad Autónoma les será aplicable la norma emanada de estas, en cambio, si desarrollan su actividad en diferentes Comunidades Autónomas les será aplicable la Ley estatal.

${ }^{21}$ De "despropósito normativo" califica la multiplicidad de normas sobre cooperativas Vicent Chuliá, 2008: 695.
} 
En los casos de fusiones dentro de una misma Comunidad Autónoma no existe ningún problema, porque la Ley aplicable es la autonómica correspondiente a la de las sociedades participantes en la operación, y en el caso de no contemplarse algún aspecto relevante, será de aplicación supletoria la LCOOP. El problema lo encontramos en los casos en los que en la fusión participan sociedades cooperativas de distintas Comunidades Autónomas (fusión supra-autonómica), porque no existe una ley armonizadora que solucione las contradicciones o discrepancias existentes entre las diferentes normas autonómicas aplicables. En estos casos son multitud de cuestiones las que se plantean: ¿Qué normativa se aplica en estas operaciones: la estatal, la de cada Comunidad Autónoma de las cooperativas participantes, la de dónde se encuentre la sociedad resultante $?^{22}$ ¿Se podría aplicar la Ley de Modificaciones Estructurales $?^{23}$ ¿Debería dictarse una nueva norma unificadora? ${ }^{24}$, etc. Aunque la doctrina se ha ocupado en ocasiones de esta problemática, pidiendo de forma generalizada una Ley armonizadora, no se ha visto satisfecha esta petición. Por todo ello, la doctrina considera complicada la fusión supra-autonómica de cooperativas, y en muchas ocasiones se huye hacia otras alternativas de integración como las cooperativas de segundo grado, la creación de grupos cooperativos o desarrollar acuerdos intercooperativos ${ }^{25}$.

\footnotetext{
${ }^{22} \mathrm{Si}$ tuviéramos que aplicar el artículo 9.11 del Código Civil que dispone que en la fusión de sociedades de distinta nacionalidad se tendrán en cuenta las respectivas Leyes personales, esto es, la determinada por su nacionalidad, o el artículo 16.1.a) del mismo cuerpo legal que se refiere a la vecindad civil cuando el conflicto de leyes se debe a la coexistencia de distintas legislaciones civiles en el territorio nacional, esto implicaría que la adopción de los acuerdos por parte de cada una de las cooperativas fusionadas (por ejemplo) se someten a la Ley, estatal o autonómica correspondiente, y los actos pertenecientes a la sociedad resultante exigirían la aplicación de las Leyes cooperativas implicadas. El problema es que faltan criterios legales para esta coordinación normativa (Paniagua, 2005). ¿No sería posible en ningún caso la aplicación de la LCOOP? Partimos del reconocimiento unánime de la LCOOP como "norma integradora, interpretativa o de aplicación supletoria" (Vicent Chuliá, 2008: 696), pero no de norma armonizadora por mucho que lo haya reclamado la doctrina, y aunque su creación parezca más una cuestión de carácter político que jurídico (Macías, 2006).

${ }^{23} \mathrm{La}$ LME tampoco parece aplicable en estos casos. El artículo 2.II LME dispone que las modificaciones estructurales de estas sociedades cooperativas, se regirán por su específico régimen legal. Quizás el régimen jurídico que contempla también debería extenderse a las cooperativas pues en lo básico (fases del procedimiento y garantía de socios y acreedores) coincide con la regulación autonómica y estatal de cooperativas.

${ }_{24} \mathrm{La}$ disparidad en la regulación autonómica de la sociedad cooperativa puede afectar al principio de unidad de mercado y al principio de igualdad, por ello, se hace necesaria la aprobación de una Ley de Armonización (León, 1999). Pero no sería suficiente con una Ley de armonización, sino que sería necesaria una regulación unitaria de las modificaciones estructurales en todo el ámbito del Derecho Societario que no sólo incluyera a las sociedades tradicionalmente consideradas mercantiles como ya hace la LME, puesto que podemos encontrarnos antes fusiones heterogéneas, por ejemplo, entre una sociedad cooperativa y una sociedad de capital. Así, las normas autonómicas que contemplan las fusiones entre cooperativas y sociedades mercantiles y civiles o la transformación en éstas, son vistas por la doctrina como una "clara extralimitación" de la competencia normativa autonómica en el ámbito civil y mercantil (Paniagua, 2005: 313 y 315).

${ }^{25}$ Esto no significa que en la práctica no existan ejemplos de fusiones supra-autonómicas. Por ejemplo, en el año 2009 se llevó a cabo una fusión en el sector arrocero entre "Arrocera del Pirineo" (cooperativa de segundo grado) y Grupo AN (también sociedad cooperativa), formando la "Cooperativa Arrocera Navarro Aragonesa" o más recientemente en 2014 la fusión entre "Baco Bodegas Asociados Cooperativas", Sdad. Coop. de C-LM y la Sdad. Coop. And. "Dcoop".
} 
Una vez realizadas estas presiones sobre el marco jurídico aplicable, podemos pasar ahora a identificar la fusión como aquel proceso por el cual dos o más sociedades que se extinguen, transmiten su patrimonio en bloque a una nueva sociedad que se constituye a estos efectos (fusión por creación de nueva sociedad), o a una de ellas (fusión por absorción), pasando generalmente sus socios a la sociedad que se constituye o a la sociedad absorbente en función del negocio que todas ellas asumen (Gadea, Sacristán, Vargas, 2009). Todas las leyes cooperativas reconocen estas dos modalidades de fusión. El régimen del procedimiento de fusión es bastante gravoso debido a los efectos que desencadena dicha operación al afectar a la estructura financiera, patrimonial y orgánica y asimismo a la posición de los socios. La fusión produce tres efectos esenciales: 1) La extinción de las sociedades que participan en la fusión, ya sea de todas ellas en la fusión por constitución de nueva sociedad, o de las sociedades absorbidas en la fusión por absorción. Aunque la mayoría de leyes cooperativas se refieran a disolución sin liquidación (por ejemplo, art. 63.2 LCOOP), debe entenderse que se refieren a extinción, pues la misma es un efecto propio de la fusión que no lleva aparejada la liquidación ${ }^{26}$. 2) La sucesión universal o traspaso en bloque del patrimonio de las sociedades que se extinguen a la sociedad resultante. A través de la transmisión por sucesión universal se consigue transmitir por un solo acto todo el patrimonio de las sociedades que se extinguen sin necesidad de cumplir las reglas de transmisión de los distintos bienes, derechos y obligaciones como se exigiría en otro caso. 3) El traspaso de los socios de las sociedades que se extinguen a la sociedad de nueva constitución o absorbente. Con el objeto de respetar la posición de los socios en las antiguas cooperativas, en el proyecto de fusión se fija la cuantía que se reconoce a cada socio como aportación en el capital de la sociedad resultante de la fusión; se reconocen sus derechos en la cooperativa resultante; etc., a diferencia de lo previsto en la LME que articula este traspaso de los socios a través de la relación de canje ${ }^{27}$.

Es posible la participación en una fusión de una cooperativa en liquidación, siempre que no haya comenzado el reembolso de sus aportaciones a los socios. También prevén la mayoría de las leyes cooperativas la posibilidad de que una cooperativa se fusione con otro tipo de sociedad (por ejemplo, una S.A., S.R.L o una S.A.T.), lo cual introduce ciertas especialidades en cuanto al régimen legal aplicable -se aplica la normativa reguladora de la

\footnotetext{
${ }^{26}$ Sobre la justificación de por qué la fusión no se engloba dentro de los procesos de disolución-liquidación puede verse Gadea, Sacristán, Vargas, 2009.

${ }^{27}$ En las cooperativas "la posición del socio no depende de la aportación patrimonial a la sociedad resultante de las sociedades cooperativas que se extinguen, sino que como consecuencia del juego de las características de derecho cooperativo el cambio de posición de socio parte de las aportaciones al capital de la cooperativa que se extingue, incrementado en su caso, con el valor correspondiente en las reservas repartibles" (Gadea, Sacristán, Vargas, 2009: 573).
} 
sociedad resultante- y en cuanto al destino de los fondos obligatorios si la sociedad resultante no es una cooperativa. La fusión heterogénea facilita la reordenación empresarial y permite adaptarse a las sociedades a las exigencias del tráfico (Gadea, Sacristán, Vargas, 2009).

Por otra parte, aunque algunos autores realizan la división en fases del procedimiento de fusión de otra forma, generalmente se distinguen cuatro fases: 1) Una primera fase previa centrada en las relaciones iniciales entre las distintas sociedades implicadas, los protocolos de fusión, a los que la ley no presta atención; y en la redacción del proyecto de fusión. Este documento es el fruto de tales negociaciones previas y del intercambio de información comercial, económica y financiera. Son los Consejos Rectores los encargados de su redacción y suscripción como convenio propio ${ }^{28}$ y sobre quiénes recae un deber de abstención de realizar cualquier clase de acto o de concluir cualquier contrato que pueda comprometer la aprobación del proyecto o modificar sustancialmente la proporción de la participación de los socios de las cooperativas extinguidas en la nueva o absorbente. Por el peso que tiene el proyecto en el procedimiento de fusión, se le ha considerado, por parte de la doctrina, como el negocio jurídico esencial de la fusión que debe ser aprobado por las Asambleas generales ${ }^{29}$. Este documento tiene que tener un contenido mínimo exigido por las leyes cooperativas ${ }^{30}$, aunque podrán incluirse cualesquiera otros puntos que los Consejos Rectores de las distintas cooperativas consideren necesarios. El proyecto deberá ser aprobado por las Asambleas generales de las cooperativas que participan en la fusión en el plazo de seis meses para que no quede sin efecto. 2) Una segunda fase gira en torno a la adopción del acuerdo de fusión por parte de las sociedades participantes en la operación, a lo que deberá preceder la convocatoria previa de las Asambleas, en la que se incluirán las menciones mínimas del proyecto, y la puesta a disposición de los socios de una serie de documentos relacionados con la fusión (proyecto de fusión, balance de fusión, informe de los Consejos Rectores, las cuentas anuales de los tres últimos ejercicios de las sociedades participantes, el proyecto de estatutos o las

\footnotetext{
${ }^{28}$ Sobre los Consejos Rectores recae el peso de esta primera fase de la fusión, aunque pueden, en cualquier momento, pedir instrucciones o consultar a los socios sobre el interés real que tienen en la fusión.

${ }^{29} \mathrm{Su}$ aprobación implica "una determinada voluntad contractual: la de continuar y, en su caso, concluir el proceso de fusión" (Morillas, Feliú, 2002: 504).

${ }^{30}$ Las menciones mínimas exigidas son las siguientes: a) La denominación, clase, domicilio de las cooperativas que participen en la fusión y de la nueva cooperativa, en su caso, así como los datos de inscripción de aquéllas en el Registro de Cooperativas; b) Sistema para fijar la cuantía que se reconoce a cada socio o asociado, en su caso, de las sociedades disueltas, como aportaciones al capital social de la sociedad cooperativa nueva o absorbente, computándose, cuando existan, las reservas voluntarias de carácter repartible; c) Los derechos y obligaciones que se reconozcan a los socios de las cooperativas extinguidas en la sociedad nueva o absorbente; d) La fecha a partir de la cual las operaciones de las cooperativas que se fusionen habrán de considerarse realizadas, a efectos contables, por cuenta de la cooperativa nueva o absorbente; e) Los derechos que, en su caso, correspondan en la sociedad cooperativa nueva o absorbente a los titulares de títulos participativos, u otros asimilables, de las cooperativas que se extingan.
} 
modificaciones pertinentes en los de la sociedad resultante, etc.). Las Asambleas generales, que son las competentes para ello, deberán aprobar el proyecto de fusión sin modificaciones puesto que sino el procedimiento debería empezar de nuevo-, por la mayoría reforzada que precise cada ley cooperativa, que generalmente es de dos tercios o tres quintos de los asistentes $^{31}$. El acuerdo una vez adoptado deberá ser publicado en el Boletín Oficial correspondiente y en un periódico de los de mayor circulación del territorio que especifique cada ley. Desde que es aprobado el acuerdo de fusión, las Asambleas generales de todas las cooperativas quedan obligadas a continuar el procedimiento de fusión. 3) En una tercera fase, después de que se haya producido la aprobación del acuerdo por parte de las Asambleas generales de todas las sociedades, se produce un periodo de pendencia de la operación, a la espera de que transcurra el tiempo legalmente previsto para que los socios disconformes con la operación puedan ejercer, en su caso, su derecho de separación - o causar baja justificada- ${ }^{32}$ y los acreedores su derecho de oposición a la fusión, con el objeto de que se les garantice o satisfaga sus créditos en el caso de estar legitimados. 4) Por último, se podrá proceder a elevar el acuerdo de fusión a escritura pública única, en la que constará el balance de fusión de las sociedades que se extingan ${ }^{33}$, y a su posterior inscripción en el Registro de cooperativas correspondiente. La eficacia de la fusión queda supeditada a esta inscripción ${ }^{34}$. Los acuerdos de fusión, a diferencia de la LME que sí prevé la posibilidad de impugnar en sí misma la fusión, serán impugnables de acuerdo con las reglas generales de impugnación de acuerdos sociales $^{35}$.

Las cooperativas que opten por esta técnica de concentración empresarial ${ }^{36}$, tienen que tener claro que se produce una integración absoluta de las entidades fusionadas, difícilmente

\footnotetext{
${ }^{31}$ Por ejemplo: exigen dos tercios los arts. 104 LCCLM, 109.1 LCPA, 64.2 LCARA, y 36.6 LCCV; y, en cambio, la mayoría de las tres quintas partes de los votos los arts. 56.1 LFCN y 33.2 LCAND.

${ }^{32}$ La cooperativa resultante de la fusión asumirá la obligación de la liquidación de las participaciones al socio disconforme con la fusión, en la forma regulada en la normativa para el caso de baja justificada.

${ }^{33}$ Además, si la fusión es por creación de una nueva sociedad, la escritura contendrá las menciones necesarias para constituir estas sociedades. En cambio, si se trata de fusión por absorción, contendrá las modificaciones estatutarias que se hubieran acordado por la sociedad absorbente con motivo de la fusión. La escritura permitirá cancelar los asientos de las sociedades extinguidas y la inscripción de la constituida o de las modificaciones de la absorbente en el Registro de Cooperativas.

${ }^{34}$ Por ejemplo: arts. 82.3 LCPV y 81.4 LCCAT.

${ }^{35}$ En concreto, la acción de impugnación de los acuerdos nulos caduca en el plazo de un año desde la fecha en que se adoptó el acuerdo o desde su inscripción. Igual, hubiera sido conveniente que el legislador hubiera previsto un plazo más breve para la impugnación de la fusión, en línea con el artículo 47 LME que lo fija en tres meses, pues un plazo tan largo para impugnar la fusión puede crear inseguridad jurídica.

${ }^{36}$ Esta técnica de concentración empresarial ha sido especialmente utilizada por las sociedades de capital, por lo que la doctrina ha tratado poco su estudio desde el punto de vista de la cooperativa (García, 2013; Juliá, 2004; o Meliá, 2014). No obstante, muchas de las consideraciones realizadas en sede de fusión de sociedades de capital pueden ser trasladables a las cooperativas (Cortés y Pérez, 2008; o Sequeira, 1993).
} 
reversible a no ser que se realice una operación de escisión. En muchas ocasiones, las fusiones fracasan porque se llevan a cabo de una forma rápida y casi improvisada para responder ante la crisis de la sociedad, tratar de frenar a un competidor, etc. Las fusiones deben ser estudiadas con detenimiento, en especial, se debe tener cuidado al escoger a la empresa con la que se quiere fusionar y fijar bien todos los aspectos de la operación y del futuro funcionamiento, gestión y organización de la sociedad resultante. En muchas fusiones se producen problemas por la actitud de los propios directivos o miembros del Consejo Rector que no quieren que su puesto se vea en peligro ante la fusión, o los propios socios no se sienten identificados en la sociedad resultante que ha podido cambiar la denominación social, haber realizado un cambio de orientación en sus políticas comerciales o de trato con los socios, etc. Por todo ello, es recomendable como paso previo a una operación de tanta envergadura, que se utilice como vía previa, alguna otra forma de integración como los grupos cooperativos o las sociedades cooperativas de segundo grado, de la que pasamos a ocuparnos a continuación. Tanto en el caso de los grupos como en las cooperativas de segundo grado, aunque las sociedades cooperativas integrantes siguen existiendo de forma independiente, se genera cierta unidad ${ }^{37}$.

\subsection{Las cooperativas de segundo grado}

La constitución de cooperativas de segundo grado responde a la necesidad de colaboración empresarial. Las cooperativas de segundo grado son "aquellas cooperativas que se componen de cooperativas de la misma o distinta clase y, en su caso, de socios de trabajo, empresarios individuales y otras entidades, con el objeto de promover, coordinar y desarrollar fines económicos comunes de los socios, y reforzar e integrar la actividad económica de los mismos" (Gadea, Sacristán, Vargas, 2009: 558). Las cooperativas de segundo grado se caracterizan por: a) mantener la forma societaria de sociedad cooperativa; b) sus socios son sociedades cooperativas y otras personas físicas o jurídicas; c) desarrollar las ideas y fines de las sociedades cooperativas socios que la integran; d) ser la cabeza de un grupo empresarial; y e) poseer personalidad jurídica independiente de las sociedades cooperativas que la forman

\footnotetext{
${ }^{37}$ Ambos fenómenos de integración (también la sociedad resultante como consecuencia de una fusión) pueden ser estudiados desde la perspectiva de posibles abusos de posición de dominio (arts. 2 LDC y 102 TFUE). Los abusos de posición de dominio están prohibidos y no admiten exención posible. Una empresa tiene una posición dominante cuando puede obstaculizar el mantenimiento de una competencia efectiva en un mercado en concreto, pudiendo actuar de forma independiente respecto a sus competidores, clientes y consumidores (por ejemplo, el establecimiento de precios abusivos muy altos impuestos a distribuidores o consumidores, o muy bajos para expulsar del mercado a los competidores; o la limitación de la producción, el mercado o el desarrollo técnico en perjuicio de los consumidores; o la imposición de contratos ligados; etc.).
} 
(Puentes, Velasco, Vilar, 2013). Las cooperativas de segundo grado ofrecen determinadas ventajas frente a otros procesos de integración (Puentes, Velasco, Vilar, 2013), como respetar los principios cooperativos; mantener la autonomía jurídica y empresarial de las sociedades que integra; determinar el grado más o menos intenso de implicación de las entidades socias; conseguir mejoras en la administración, producción y comercialización; su constitución conlleva menos gastos que otras formas de integración, etc. Pero, a la misma vez, también pueden surgir ciertas dificultades (Puentes, Velasco, Vilar, 2013), como la adopción de una democracia delegada (las sociedades participan a través de los representantes elegidos, pero no participan de forma directa los socios de las mismas); se requiere un personal altamente cualificado para dirigir la sociedad, de lo que en muchos casos se carece; etc.

Las cooperativas de segundo o ulterior grado requieren un mínimo de dos cooperativas de grado inmediatamente inferior para constituirse. Si las sociedades cooperativas de segundo o ulterior grado están formadas únicamente por sociedades cooperativas serán homogéneas aunque también podrán integrarlas socios de trabajo, SAT y empresarios individuales-; y si están integradas por otras entidades susceptibles de ser socias serán heterogéneas. La mayoría de socios y de votos sociales deben corresponder a las sociedades cooperativas, a excepción de los socios de trabajo, con lo cual se asegura que el control de la sociedad pertenezca a las cooperativas. En cualquier caso, las aportaciones de cada socio al capital de las cooperativas de segundo o ulterior grado tampoco podrán sobrepasar ciertos límites totales (respecto al total de la sociedad) ni relativos (en comparación con los poseídos por cooperativas). Si se produce la baja de alguna de las cooperativas socias, en el caso de que fueran solamente dos, supondrá la disolución de la cooperativa de segundo grado o su transformación en cooperativa de primer grado (Gadea, Sacristán, Vargas, 2009). Aparte, las cooperativas de segundo grado puedan transformarse en cooperativas de primer grado, siendo absorbidas las cooperativas socias, que podrán ejercer si están disconformes su derecho de separación ${ }^{38}$.

El régimen legal de las cooperativas de segundo grado es muy similar al de las de primer grado, puesto que las leyes cooperativas sólo prevén determinadas particularidades, que deben ser completadas por lo establecido en los Estatutos y en el Reglamento de Régimen Interno, siendo para lo demás aplicable lo previsto de forma general en las leyes cooperativas, en cuanto la específica función y naturaleza de las cooperativas de segundo o ulterior grado lo

\footnotetext{
38 "Se trata de un proceso peculiar de modificación estructural cooperativa, que implica, simultáneamente, la transformación o conversión de la cooperativa de segundo grado en cooperativa de primer grado, sin previa disolución y sin creación de una personalidad jurídica nueva” (Valiñani, Aizega, 2003: 16).
} 
permitan (Alfonso, 2000; o Vázquez, 2002). Por ejemplo, se establecen ciertas especialidades en cuanto a la organización de la Asamblea General o el Consejo Rector ${ }^{39}$. En los Estatutos deben recogerse las facultades esenciales que deben ser transferidas a los órganos de dicha cooperativa, por ser necesarias para el desarrollo de su objeto social. Asimismo, si la cooperativa se constituye con fines de integración empresarial, los Estatutos habrán de determinar las áreas de actividad empresarial integradas, las bases para el ejercicio de la dirección unitaria del grupo y las características de éste. Además, los estatutos regularán las materias o áreas respecto de las cuales las propuestas de las entidades asociadas serán meramente indicativas, y no vinculantes, para la cooperativa de segundo o ulterior grado.

Si se disuelve la cooperativa de segundo grado, el haber líquido resultante se distribuirá entre los socios en proporción al importe del retorno percibido en los últimos cinco años o, en su defecto, desde la constitución de aquella. En el caso de las sociedades cooperativas socias, se destinará siempre al Fondo de Reserva Obligatorio de cada una de ellas. Por último, se considerarán resultados cooperativos los retornos que reciban las cooperativas socias de las de segundo o ulterior grado, al igual que los intereses que se devenguen por sus aportaciones al capital y los rendimientos derivados de la financiación voluntaria.

\subsection{Los grupos cooperativos}

El grupo de sociedades es una técnica de organización de empresas, cuya regulación es escasa, limitándose poco más de a lo que prevé el artículo 42 del Código de Comercio, el artículo 18 de la Ley de sociedades de capital y las previsiones que han realizado las normas autonómicas y la estatal de cooperativas ${ }^{40}$. En el grupo la titularidad jurídica de la empresa está repartida en varios sujetos que, aunque formalmente autónomos, realmente responde a las directrices marcadas por la dirección económica del grupo (Alfonso, 2006). Un grupo cooperativo está formado por varias sociedades cooperativas, en el que existe una cabeza de grupo que ejercita facultades o emite instrucciones de obligado cumplimiento para las

\footnotetext{
39 En la Asamblea General de las cooperativas de segundo grado, las cooperativas miembros estarán representadas por su Presidente o por el socio de la misma que se designe por su Consejo Rector, sin poder delegarse la representación a favor de otra de las cooperativas asociadas; la composición cuantitativa del Consejo Rector podrá fijarse estatutariamente mediante el establecimiento de un número mínimo y otro máximo; etc.

${ }^{40}$ Gran parte de las Leyes autonómicas de cooperativas, junto a la estatal, dedican un artículo expresamente a los grupos cooperativos. Podemos tomar de ejemplo, por lo que respecta a la normativa autonómica, los artículos 109 LSCA, 125 LCCAT, 123 LCCV y 135 bis LCPV.
} 
cooperativas agrupadas, y conlleva una unidad de decisión (art. 78.1 LCOOP) ${ }^{41}$. Las leyes cooperativas que regulan esta figura, reconocen la posibilidad de que las cooperativas puedan asociarse entre sí y con otras entidades para formar un grupo cooperativo. En el concepto de grupo existen tres elementos necesario: 1) elemento subjetivo: la pluralidad de personas; 2) elemento finalista: la dirección unitaria; y 3) elemento instrumental: un medio que pone en conexión los otros dos elementos, como puede ser la forma contractual en los grupos por coordinación.

Por otra parte, podemos diferenciar entre grupos cooperativos propios e impropios ${ }^{42}$. Los grupos cooperativos propios o grupo subordinado ${ }^{43}$ son aquellos en los que existe una sociedad cabeza de grupo que ejercita facultades o emite instrucciones de obligado cumplimiento para el grupo, pudiendo ser o no dicha cabeza de grupo una cooperativa, pero en el caso de que no lo sea, los miembros del grupo han de ser en su mayoría sociedades cooperativas. $\mathrm{Y}$ es que en principio una sociedad cooperativa sólo puede participar en un grupo por subordinación como sociedad dominante, porque si no se iría en contra del principio democrático. Los grupos por subordinación tienen una estructura jerárquica de dependencia, ejerciendo la sociedad matriz el papel de sociedad dominante, la cual controla al resto de sociedades mediante diferentes técnicas societarias (participación en el capital de las otras sociedades, derecho de voto, etc.). Por el contrario, se entiende por grupo cooperativo impropio o grupo paritario o por coordinación, aquel en el que sus miembros, que habrán de ser sociedades cooperativas en su mayoría, se articulan en un plano de igualdad, funcionando sobre la base de un principio de coordinación. La dirección unitaria no se impone por una entidad superior sino que la gestión del grupo se caracteriza por una coordinación horizontal a través de acuerdos de colaboración mutua entre las cooperativas miembros (Gadea, Sacristán, Vargas, 2009), y puede conseguirse mediante la constitución de un órgano o una sociedad a la que se le atribuya el ejercicio de la misma o la creación de otros vínculos ${ }^{44}$. Esta clase de

\footnotetext{
${ }^{41}$ Con el grupo se pretende la sumisión consensuada de las sociedades partícipes a una dirección unitaria y común como vía para coordinar sus actividades empresariales (Alfonso, 2006).

${ }^{42}$ Podemos diferenciar entre distintos tipos de grupos de sociedades: grupos de derecho o de hecho; grupos de base societaria, de base contractual o de base personal; grupos de actividad homogénea y grupos de actividad heterogénea; grupos industriales y grupos financieros; grupos centralizados y grupos descentralizados; y grupos por subordinación y grupos por coordinación. Realiza una clasificación minuciosa De Arriba, 2004.

${ }^{43}$ Presta mayor atención a la distinción entre grupos subordinados y por coordinación De Arriba, 2004.

${ }^{44}$ Existen diversas formas de conseguir esa dirección unitaria que caracteriza a los grupos paritarios: 1) una de las sociedades agrupadas pueden ejercer el papel de "entidad cabeza del grupo", sociedad que estaría al mismo nivel que el resto, pero que se le asignan una serie de competencias y funciones por razones de agilidad y de mejor funcionamiento de la dirección del grupo. Para asegurar la participación del resto de entidades en la formación de la dirección unitaria se utilizan las interdependencias personales, es decir, que las personas que forman parte de los órganos de administración de las sociedades agrupadas participen en esta sociedad o
} 
grupo se adecua mejor a la naturaleza y características de las cooperativas, al respetar los principios de igualdad y cooperación. El grupo podría representarse "como una pirámide invertida" (Gadea, Sacristán, Vargas, 2009: 553) en la que la sociedad cabeza de grupo se posiciona en un escalón inferior en la base, y arriba las sociedades participantes.

Las entidades que integran el grupo deben ser cooperativas, aunque la sociedad que ejerza la función de "cabeza de grupo" puede tener otra naturaleza (sociedad anónima, sociedad de responsabilidad limitada, sociedad civil, sociedad agraria de transformación, asociación, etc.), ya que, generalmente, las leyes cooperativas no precisan lo contrario ${ }^{45}$. Las entidades que forman el grupo paritario deben prestar su consentimiento a la política general del mismo y pueden oponerse a ella si no se adecúa a sus intereses. El instrumento jurídico idóneo para formar un grupo por coordinación es el contrato de organización, en virtud del cual, dos o más entidades independientes entre sí acuerdan someter la gestión de sus sociedades a una dirección unitaria común y coordinada (De Arriba, 2004). Será necesario que la Asamblea general (u órgano equivalente) de las entidades aprueben la incorporación al grupo. En aplicación del principio cooperativo de puertas abiertas, las cooperativas miembro podrán separarse del grupo en cualquier momento (Embid, 2003).

El interés de cada cooperativa se ordena al interés del grupo ${ }^{46}$, pues ambos pretenden la consecución de un objetivo común a todas las sociedades. Por este motivo, el interés del grupo debería prevalecer sobre los intereses particulares de cada sociedad, siempre que se prevea alguna forma de tutela que proteja los intereses de los posibles perjudicados. Por otra parte, el poder de dirección no es ilimitado, sino que encuentra sus límites en el respeto de los principios cooperativos, la ley, lo establecido en el contrato de grupo y el interés del grupo. La emisión de instrucciones pueden ser en los ámbitos de gestión, administración o gobierno, entre lo que se pueden incluir: a) establecimiento en las cooperativas de base de normas estatutarias y reglamentarias comunes; b) establecimiento de relaciones asociativas entre las entidades de base; c) compromisos de aportación periódica de recursos calculados en función de su respectiva evolución empresarial o cuenta de resultados (art. 78 LCOOP). Los compromisos generales asumidos ante el grupo deben formalizar por escrito. Puede ser en los

participen en el órgano de gestión de la sociedad directora. 2) La creación ad hoc de una sociedad directora del grupo paritario, en la que participarán todas las sociedades del grupo. 3) Crear un órgano sin personalidad jurídica que tenga la misión de dirigir el grupo (consejo del grupo, órgano común, comité de dirección, etc.), que puede ser acogido en el seno de cualquiera de las sociedades participantes.

${ }^{45}$ Los arts. 125.1 LCCAT y 135 bis.1 LCPV sí que exigen que la entidad cabeza de grupo sea una cooperativa.

46 Éste se ve también limitado en su actuación por el principio de proporcionalidad y el de supervivencia económica de las sociedades participantes. 
estatutos de la entidad cabeza de grupo o mediante otro documento contractual, que se eleve a escritura pública, debiendo incluir la duración -si es limitada-, el procedimiento para la modificación, el procedimiento para la separación de una entidad del grupo, y las facultades que se atribuyan a la entidad cabeza de grupo. El acuerdo de integración en un grupo deberá anotarse en la hoja correspondiente a cada sociedad cooperativa en el Registro competente.

La responsabilidad derivada de las operaciones que realicen las entidades miembros del grupo cooperativo directamente con terceros no se extenderá al grupo ni al resto de sus integrantes (art. 78.6 LCOOP), lo cual es consecuencia de la falta de personalidad jurídica del grupo. Dicha afirmación debería matizarse en ciertos casos, y realizarse el "levantamiento del velo", pues podría probarse que la conducta de una de las sociedades integrantes en el grupo no le es imputable de forma aislada, sino también al resto de sociedades que forman el grupo, incluida la matriz o cabeza de grupo (Alfonso, 2006).

\section{CONCLUSIONES}

Parece clara la necesidad de que las cooperativas y otras entidades asociativas de carácter agroalimentario ganen dimensión para ser más competitivas y tengan mayor poder de negociación frente al resto de eslabones de la cadena alimentaria. Las distintas vías de integración difieren en nivel de intensidad, en costes y reticencia de los cooperativistas. La fusión posibilita alcanzar una mayor dimensión a las empresas, que supone el grado máximo de concentración, al integrarse una de las cooperativas en la otra sociedad o constituyéndose una nueva, pero se trata de un procedimiento complejo, lento y costoso. Los grupos cooperativos y las cooperativas de segundo grado son formas de integración menos intensas y más flexibles, que permiten coordinar el funcionamiento independiente de las cooperativas al fijar un interés común que contribuya a alcanzar mayores logros que repercutieran positivamente en las cooperativas miembros. A pesar del complejo marco normativo con el que contamos en España, en los aspectos esenciales el régimen de la fusión y las otras vías de integración coinciden en las distintas normas de cooperativas, por lo que no debe ser un grave impedimento para llevarlas a cabo.

La LFIC tiene un objetivo ambicioso y claro: conseguir esa mayor dimensión de las cooperativas y otras entidades asociativas agroalimentarias. Pero no basta con buena voluntad, sino que es necesario ofrecer de verdad unas medidas de fomento de las operaciones de integración suficientes y adecuadas para alcanzar tal objetivo. Debería haberse 
aprovechado en su momento la ocasión para haber realizado modificaciones de calado en la regulación de las cooperativas y en materia fiscal (Consejo Económico y Social, 2013). No es suficiente con conceder un premio - preferencia en subvenciones y ayudas- a las entidades que consigan tal dimensión, sino que lo debería haberse hecho era allanar el camino para conseguir ese tamaño de las cooperativas que les permitan ser altamente competitivas. En concreto, podría haberse aclarado qué régimen es el aplicable a las fusiones entre cooperativas regidas por distintas leyes autonómicas (fusiones supra-autonómicas), cuál es el régimen supletorio en el caso de silencio legal en la regulación de algún aspecto de estas operaciones, etc. Incluso, podrían concederse mayores incentivos, no sólo de carácter económico, a las sociedades que se fusionen, constituyan cooperativas de segundo grado o grupos cooperativos, sino sobre todo en forma de asesoramiento jurídico y organizativo sobre cómo realizar tales integraciones. Por otra parte, con los umbrales fijados por el REAP y con el requisito de tener un ámbito de actuación supra-autonómico, va a ser difícil que muchas cooperativas $\mathrm{u}$ otras entidades obtengan el reconocimiento como Entidades Asociativas Prioritarias. Esto va a provocar que las distintas Comunidades Autónomas creen una figura paralela de carácter regional, como ya ha ocurrido en Castilla y León. En cualquier caso, con la LFIC se demuestra que la integración de cooperativas y otras entidades agroalimentarias no es sólo una tendencia empresarial, sino que es además una política pública fomentada por los poderes públicos (Palma, 2013).

\section{BIBLIOGRAFÍA}

ALFONSO SÁNCHEZ, R. (2000) La integración cooperativa y sus técnicas de realización: la cooperativa de segundo grado. Valencia: ed. Tirant lo Blanch.

-(2006) Grupos y alianzas de sociedades. Especial referencia al grupo cooperativo y a la cooperativa de segundo grado. En: PULGAR EZQUERRA, J. (dir.) Cooperativas agrarias y sociedades agrarias de transformación. Madrid: Dykinson, pp. 727-780.

- (2010) Respuesta del ordenamiento jurídico español ante la realidad de la Sociedad Cooperativa Europea. CIRIEC-España. Revista jurídica de economía social y cooperativa, № 21, pp. 169-198.

ARCAS LARIO, N. (2008) La Sociedad Cooperativa Europea como forma de concentración empresarial. En: ALFONSO SÁNCHEZ, R. (dir.) La Sociedad Cooperativa Europea Domiciliada en España. Cizur Menor (Navarra): ed. Thomson Aranzadi, pp. 57-95. 
CALVO CARAVACA, A.L.; CARRASCOSA GONZÁLEZ, J. (2003) Mercado único y libre competencia en la Unión Europea. Madrid: Ed. Colex.

CALVO VÉRGEZ, J. (2013) La nueva Ley 13/2013 y la creación de la figura de la 'entidad asociativa prioritaria': un paso adelante en la competitividad de las cooperativas agrarias. Actualidad Jurídica Aranzadi, № 871, p. 6.

CONSEJO ECONÓMICO Y SOCIAL (2013) Fomento de la integración de cooperativas y de otras entidades asociativas de carácter agroalimentario. Cauce: Cuadernos del Consejo Económico y Social, № 22, p. 102-105.

CORTÉS DOMINGUEZ, L.J.; Y PÉREZ TROYA, A. (2008): Fusión de Sociedades. En: URÍA, R.; MENÉNDEZ, A.; OLIVENCIA, M. (dir.). Comentario al Régimen Legal de las Sociedades Mercantiles. Tomo IX, Vol. 2.․ Cizur Menor (Navarra): ed. ThomsonCivitas.

DE ARRIBA FERNÁNDEZ, M. L. (2004) Derecho de Grupo de Sociedades. Madrid: ed. Thomsom Civitas.

EMBID IRUJO, J.M. (1991) Concentración de empresas y Derecho de cooperativas. Murcia: ed. Universidad de Murcia, Murcia, 1991.

-(2003) Introducción al derecho de los grupos de sociedades. Granada: ed. Comares.

GADEA, E.; SACRISTÁN, F. \&VARGAS VASSEROT, C. (2009) Régimen Jurídico de la Sociedad Cooperativa del Siglo XXI. Madrid: ed. Dykinson.

GARCÍA SANZ, A. (2013) Modificaciones Estructurales. En: PEINADO GRACIA, J. I. (dir.). Tratado de Derecho de Cooperativas. Vol. I. Valencia: Tirant lo blanch, pp. 780795.

JULIÁ, J.F.; SERVER, R.J.; MELIÁ, E. (2004) Los procesos de fusión en cooperativas agrarias. Manual de procedimiento. Madrid: ed. Mundi-Prensa.

LAMBEA RUEDA, A. (2005) Criterios orientativos para optar a la calificación de sociedad cooperativa europea. REVESCO. Revista de Estudios Cooperativos, № 87, pp. 77-105.

LEÓN SANZ, F.J. (1997) Fusión, transformación y otras modificaciones estructurales de sociedades cooperativas. Revista de Derecho de Sociedades, № 9, pp. 25-60.

MACÍAS RUANO, A.J. (2006) Modificaciones estructurales en las cooperativas y sociedades agrarias de transformación. En: Cooperativas agrarias y sociedades agrarias de transformación. Madrid: Dykinson, pp. 679-726.

MARTÍNEZ SEGOVIA, F.J. (2003) Primera aproximación al Estatuto de la Sociedad Cooperativa Europea. REVESCO: revista de estudios cooperativos, $\mathrm{N}^{\circ}$ 80, pp. 61-106. 
MELIÁ, E.; Y MARTÍNEZ, A.Ma. (2014) Caracterización y análisis del impacto de los resultados de las fusiones de cooperativas en el sector agroalimentario español. Almería: ed. Universidad de Almería.

MORILLAS JARILLO, M.J. \& FELIÚ REY, M.I. (2002) Curso de cooperativas. Segunda edición. Madrid: Ed. Tecnos.

PALMA FERNÁNDEZ, J.L. (2013) Fusiones de entidades agrarias: sobre la Ley 13/2013, de 2 de agosto, de fomento de la integración de cooperativas y de otras entidades asociativas de carácter agroalimentario. Noticias Breves Gómez-Acebo \& Pombo, $\mathrm{N}^{\mathrm{o}}$ septiembre.

PANIAGUA ZURERA, M. (2005) La sociedad cooperativa. Las sociedades mutuas y las entidades mutuales. Las sociedades laborales. La sociedad de garantía recíproca. Vol. I. En: OLIVENCIA, M.; FERNÁNDEZ-NOVOA, C. \&JIMÉNEZ DE PARGA, R. (dir.) Tratado de Derecho Mercantil. Madrid: ed. Marcial Pons.

PARRA DE MAS, S. (1974) La integración de la Empresa Cooperativa (Evolución de los Principios Cooperativos). Madrid: ed. de Derecho Financiero.

PASTOR SEMPERE, C. (2009) La Sociedad Cooperativa Europea domiciliada en España. REVESCO: revista de estudios cooperativos, $\mathrm{N}^{\circ}$ 97, pp. 117-144.

PAZ CANALEJO, N. (2004) Los acuerdos intercooperativos en el Derecho vigente (estatal y autonómico). Revista Jurídica del Notariado, N 52, pp. 137-209.

PUENTES POYATOS, R.; VELASCO GÁMEZ, M. DEL M. \& VILAR HERNÁNDEZ, J. (2010) Las sociedades cooperativas de segundo grado como instrumento de cooperación entre cooperativas: aspectos económicos y organizativos. Revista de Estudios Empresariales. Segunda época, № 1, pp. 103-128.

SÁNCHEZ PACHÓN, L.A. (2011) Los acuerdos intercooperativos. Un instrumento jurídico para la colaboración en momento de crisis económica. CIRIEC-España. Revista jurídica de economía social y cooperativa, $\mathrm{N}^{\mathrm{o}} 22$, pp. 121-149.

SEQUEIRA MARTÍN, A. (1993) Transformación, Fusión y Escisión. En: SÁNCHEZ CALERO (dir.). Comentarios a la Ley de Sociedades Anónimas, T. VII. Madrid: Edersa.

VALIÑANI GONZALEZ, E.; AIZEGA ZUBILlAGA, J. M. (2003) Las Cooperativas de segundo grado, grupo cooperativo y otras formas de colaboración económica. REVESCO. Revista de estudios cooperativos, $\mathrm{N}^{\mathrm{0}} 79$, pp. 7-34.

VARGAS VASSEROT, C. (2012) Las Sociedades Agrarias de Transformación. Empresas agroalimentarias entre la economía social y la del mercado. Madrid: Dykinson. 
-(2015) El gobierno corporativo y la sociedad cooperativa europea. En: Martínez-Echevarría (dir.). Gobierno Corporativo: la estructura del órgano de gobierno y la responsabilidad de los administradores. Cizur Menor (Pamplona): Aranzadi, pp. 1065-1093.

VÁZQUEZ PENA, M.J. (2002) Las cooperativas de segundo grado: peculiaridades societarias. Valencia: ed. Tirant lo Blanch.

VICENT CHULIÁ, F. (2003) La Sociedad Cooperativa Europea. CIRIEC: Revista Jurídica de Economía Social y Cooperativa, No 14, pp. 51-82.

-(2008) Introducción al derecho mercantil. Valencia: Tirant lo Blanch. 